\title{
APLICACIONES EDUCATIVAS DE LOS VIDEOJUEGOS: EL CASO DE WORLD OF WARCRAFT
}

\section{EDUCATIONALAPPLICATION OF VIDEOGAMES: THE CASE OF WORLD OF WARCRAFT}

\author{
Alodia Quesada Bernaus \\ alodia.quesada@gmail.com \\ Dr. Santiago Tejedor Calvo \\ santiago.tejedor@uab.cat
}

\begin{abstract}
Universidad Autónoma de Barcelona. Facultad de Ciencias de la Comunicación. Departamento de Periodismo. Edificio I, despacho 49, 08193, Campus UAB, Cerdanyola del Vallès (España)
\end{abstract}

Los videojuegos se han convertido en uno de los productos de ocio y entretenimiento de mayor alcance en los últimos años, superando a la industria del cine y la música. Sin embargo, los estudios desarrollados en torno a las posibilidades educativas de los mismos han sido muy escasos y se han focalizado en un conjunto determinado de productos. En este artículo, se presentan los resultados de una investigación de un año de duración centrada en el videojuego World of Warcraft. La metodología de la investigación se ha caracterizado por la combinación de diferentes técnicas y aproximaciones al objeto de estudio planteado. Por un lado, para el desarrollo del citado estudio, se produjo un documental sobre el videojuego, posteriormente, se procedió al análisis del producto audiovisual, enriquecido con un intercambio de cuestionarios con expertos y usuarios del videojuego. Además mediante 220 encuestas a jugadores y ex jugadores; entrevistas a los protagonistas del documental y entrevistas con expertos, se analizan las potencialidades formativas de los videojuegos. En el artículo se presenta el desarrollo metodológico junto a una serie de evidencias que permiten concluir que existe una educación a través de este tipo de videojuegos -todavía por explotar-con amplias posibilidades especialmente en lo relativo a la adquisición de competencias digitales, la promoción de la alfabetización digital y al desarrollo emocional de los jugadores.

Palabras clave: Videojuegos, educación, alfabetización digital y mediática, comunicación.

Video games have become the most diffused entertainment and leisure products in recent years, surpassing the film industry and music. Nevertheless, there are a few studies about the educational possibilities of videogames and they focus only on particular products. In this article, we presented the results of a one year research focused on the game World of Warcraft. The research methodology is characterized by the combination of different techniques and approaches: on the one hand, a documentary about the game has been produced, then proceeded to the analysis of audiovisual product, enriched with an exchange of questionnaires with experts and users of the game. Besides surveys by 220 players and former players; interviews with the protagonists of the documentary and interviews with experts, the educational potential of video games are analyzed. The article presents the methodological development with a range of evidence to conclude that there can be education through this type of game-wide possibilities to be explored- especially relating to the acquisition of digital skills, promoting digital and emotional literacy player development.

Key words: videogames, education, media literacy, communication. 


\section{Introducción.}

Los videojuegos se han convertido en un entretenimiento que ofrece una puerta de entrada del mundo digital para sus usuarios, como indica Gee (2003). Este aprendizaje ofrece a las nuevas generaciones la adquisición de competencias y la oportunidad de alfabetizarse digitalmente a través de lo lúdico. Las preocupaciones que hasta al momento tienen los educadores sobre el juego se han centrado en diferentes aspectos relativos al control como la tipología de los contenidos y el número de horas de juego. De este modo, se dejan de lado las cualidades que los videojuegos ofrecen en cuanto a términos educativos.

Desde la aparición de Pong (primer videojuego comercial de entretenimiento electrónico creado en 1972 por Atari), las formas de jugar digitalmente han evolucionado de forma considerable. Atrás han quedado los microprocesadores de los años 60 y los videojuegos de la década de los 70. Veinte años han tenido que pasar para la extensión masiva de los videojuegos y situarlos donde los encontramos hoy en día: formas de juego a través de internet que captan seguidores y permiten a los usuarios jugar desde cualquier punto geográfico en red.

Con una conexión a internet actualmente se pueden participar en videojuegos que conectan a jugadores de todo el mundo. Los entornos digitales han ido adaptándose para facilitar la creación de comunidades virtuales que viven en un mundo paralelo al real. La investigadora Silva (2010) afirma que podemos entender los medios de comunicación como prolongaciones del hombre, y califica la cibersociedad como un escenario privilegiado donde observar la interconexión de esta nueva cultura tecnológica.

Los videojuegos forman parte de esta nueva cultura tecnológica en el ciberespacio $y$ han sido estudiados como una nueva forma de socialización como señalan los trabajos de (Echevarría, 2000; Castells, 2003; Gómez, 2001; Joyanes, 1997).

En el ciberespacio se pueden realizar muchas actividades, una de ellas son los videojuegos MMORPG (Massive multiplayer online role playing game), como World of Warcraft, donde la cibersociedad y la realidad virtual ofrecen a sus usuarios un nuevo escenario digital con grandes oportunidades expresivas gracias a las nuevas tecnologías. Esta particular realidad virtual es el resultado de la creación por parte de comunidades virtuales, descritas como ecosistemas de subculturas y grupos espontáneamente constituido donde cada uno es un experimento social que nadie planificó (Rheingold, 1996).

El mundo de los videojuegos nos sitúa en un escenario digital que desarrolla una realidad virtual paralela al mundo real y donde se crean comunidades, familias virtuales que evolucionan, crecen o disminuyen como las de la vida real. Se trata de una nueva forma de socialización en red (Castells, 2003) que permite unas relaciones entre los jugadores a través de códigos de comportamientos, reglas $\mathrm{y}$ formas de vida que hacen surgir una nueva identidad, un «yo online» (Muros, 2011). La construcción de una identidad virtual, que se crea a partir de un apodo y de un clan, ofrece la posibilidad de ser otra persona para los jugadores y convivir con el resto de usuarios en los entornos virtuales. Se trata de escenarios y espacios donde se desarrollan relaciones sociales, aspectos educativos y culturales, donde los jugadores pueden 
experimentar y crear identidades que promueven la creatividad y el aprendizaje informal. Estos particulares escenarios van más allá del propósito del entretenimiento y, cada vez más, están siendo usados en el aprendizaje.

Los investigadores Thomas y Brown (2007) introducen el concepto «juego de la imaginación». Se trata de un aprendizaje diferente al estándar de los sistemas pedagógicos y que ocurre en los mundos virtuales. Los autores defienden que la imaginación juega un papel esencial en el proceso de enseñanza de los videojuegos y que existen tres aspectos de los MMORPG que los convierten en un nuevo escenario de aprendizaje: 1) los mecanismos de juego, 2) los avatares y 3) la complejidad cultural, económica y social. En la presente investigación se planteó ir más allá de la diversión del videojuego analizando que hay «además de»y «fuera de», buscando un valor educativo de este tipo de videojuegos no creados para la educación. Autores como (Gil \& Vida, 2007; Gee, 2003) ya investigaron algunos de los aspectos educativos de los juegos. Este último enumera 36 formas de aprender de un videojuego como por ejemplo: aprendizaje a partir de los errores, desarrollo de habilidades personales, emocionales y cognitivas o aprender un nuevo alfabetismo, entre otros.

Un término clave de este artículo es la palabra: videojuego. Aranda y Sánchez (2009) los definen como un recurso cultural, una herramienta básica de socialización y aprendizaje que aporta habilidades y competencias a los usuarios. Los videojuegos, según estos autores, son sistemas basados en reglas que obligan a los jugadores a entender el mundo y tomar decisiones, reglas que las vas descubriendo a medida que avanzas en el juego y, lo más importante, que se intentan romper. En los videojuegos esto no supone paralizar el juego, sino que añade un punto de emoción, diversión y complejidad. Además existen unos objetivos que se han de cumplir para avanzar: llegar a la meta, matar al monstruo... todos ellos con esfuerzo e interacción con los otros jugadores. Los otros usuarios son la ficha clave para progresar, son los protagonistas para que el juego evolucione y siga existiendo. Y es que además se establece un vínculo emocional que traslada a los videojuegos a algo más que máquinas de entretenimiento, los transforma en elementos culturales que los jugadores utilizan para divertirse, relacionarse, aprender y expresarse.

Los videojuegos pueden contribuir con el desarrollo de contenidos, de capacidades organizacionales y mejorar las habilidades y destrezas de los usuarios (Haste, 2010). Es interesante conocer el juego para saber la importancia del aprendizaje para «ver» y llegar a entender el juego como un sistema diseñado (Gee, 2003), de aquí que se desconozca o infravalore la magnitud de lo que supone jugar a este tipo de videojuegos.

McGonigal (2011) relaciona el concepto de «productividad feliz» con los videojuegos. En este caso, analizamos World of Warcraft, el videojuego más exitoso de su género MMORPG, donde millones de jugadores luchan por conseguir sus objetivos. Según McGonigal los videojuegos nos hacen felices porque nos hacen trabajar duro, porque para conseguir nuestros objetivos y cumplir los retos debemos luchar siempre de forma libre, sin imposiciones. Esta es la clave, según este autor, para alcanzar la «productividad feliz» antes citada. Marqués (2001) argumenta que los videojuegos nos atraen porque proponen 
un reto, porque reeducan a los usuarios y les hacen disfrutar, además de añadir un aliciente de control en el que los usuarios son los únicos que manejan a su personaje, sintiéndose poderosos por cumplir expectativas que en la vida real no pueden existir.

Maÿra (2013) va más allá, y habla de la importancia de incluir los videojuegos dentro de una alfabetización mediática. Propone que la media literacy incluya los videojuegos dentro de su ámbito de estudio de la alfabetización digital y mediática como una competencia. La alfabetización digital y mediática es una competencia clave en los sistemas educativos reconocida universalmente. Como la define Pérez y Varis (2012) es «el proceso de asumir y usar los códigos implicados en el sistema mediático contemporáneo, así como las habilidades prácticas necesarias para un uso adecuado de los sistemas tecnológicos en que los códigos se basan» (p.7). Y también es «la capacidad de acceder, analizar y evaluar el poder de las imágenes, sonidos y mensajes con que nos enfrentamos cada día y que juegan un papel importante en la cultura contemporánea» (p.7).

Además, los propios diseñadores de videojuegos opinan que «aprender» es la droga para captar a los jugadores (Koster, 2004), es decir, la adquisición de nuevas habilidades es lo que da el éxito a los videojuegos, los jugadores se mantienen en los juegos porque ven que avanzan jugando, que aprenden nuevas habilidades de sus personajes que les hacen seguir adelante en la historia del videojuego.

Otros diseñadores como Salen y Zimmerman (2003) opinan que los jugadores aprenden a entender y explorar sistemas complejos a través de los videojuegos, convirtiéndose estos en un medio poderoso que más allá de su objetivo principal de entretener.

Los videojuegos se han convertido en uno de los mercados más rentables superando al cine y la música. En 2013 el valor del consumo de los videojuegos en España estuvo en 762 millones de euros, frente a 691millones de la industria del cine (AEVI). En 2014 la venta de videojuegos en España alcanzó los 996 millones de euros (605 en videojuegos y 391 millones en venta de consolas) según un informe de la Asociación Española de Videojuegos (AEVI). Mientras que, según datos ofrecidos por Rentrak, lo recaudado por la taquilla de cine ha sido de 522 millones de euros. Se trata de un sector con una oferta muy amplia.

Se debe diferenciar entre videojuegos educativos, también denominados serious games (Abt,1987) y videojuegos comerciales, los cuales no están diseñados para la educación. Los denominados serious games se podrían definir como videojuegos con una intención clara educativa pero diseñados para que tengan tanto éxito como los juegos comerciales. Tienen un propósito que vas más allá del entretenimiento y el objetivo propio del mismo juego. Son videojuegos que tratan de influir en los pensamientos y acciones de los jugadores en las situaciones de su vida real. Por lo tanto, la capacidad educativa que pueden llegar a tener los videojuegos, incluso aquellos que no han sido diseñados con este fin, como es el caso de World of Warcraft, resulta sorprendente. Los expertos señalan que este tipo de videojuegos puede permitir que los usuarios aprendan y desarrollen habilidades aplicables en la vida real, sobretodo en aspectos como la coordinación motriz, la inteligencia emocional, la adquisición de habilidades y 
competencias digitales, la creatividad y la imaginación. Este conjunto de tipos de aprendizajes que están relacionados con la teoría de las inteligencias múltiples de Gardner (1993), una teoría que se origina basándose en la capacidad de las personas de resolver problemas, como hacen los jugadores de videojuegos.

\subsection{World of Warcraft.}

World of Warcraft (WoW) es considerado el videojuego en línea con mayor registro de usuarios, con 10 años de experiencia cuenta con más de 10 millones de usuarios y mantiene el Récord Guiness para el MMORPG más popular. Se trata de un videojuego comercial de rol, es decir, un juego en el que el jugador elije a su personaje lo caracteriza a su gusto y le pone un apodo o nickname. Los argumentos de este tipo de videojuegos se inspiran en la edad media y los personajes son fantásticos y en un mundo imaginario lleno de animaciones gráficas. Se trata de un entorno virtual, que tal y como Aarseth (2003) los define, poseen una dimensión experiencial (game-play), en la que los jugadores establecen sus experiencias y estrategias, una estructura (game-estructure), porque se trata de un videojuego que tiene reglas y éstas permiten progresiones a sus jugadores, $\mathrm{y}$ exploración (game-world), porque como indica el nombre de nuestro videojuego los jugadores se introducen en el mundo de Warcraft.

Este videojuego, que es el principal objeto de estudio, no es un videojuego diseñado para la educación. Sus diseñadores lo crearon básicamente con fines de entretenimiento pero, con el enfoque adecuado puede convertirse en un videojuego muy enriquecedor a nivel educativo para sus usuarios. El videojuego World of Warcraft tiene una alcance mundial muy amplio. Se trata de uno de los MMORPG con más repercusión mundial, cuenta con 1200 millones de dólares de ingresos anuales, 16 gigabytes de espacio en el disco duro, millones de horas jugadas, 6 expansiones, una extensión virtual de terreno incontrolable, una película en desarrollo dirigida por Sam Raimi prevista para 2016, un episodio de los Simpsons, uno de South Park y aparición en la serie The Big Bang Theory. En 2014, la empresa creadora, Blizzard, celebró su décimo aniversario, sacando a la luz el documental «World of Warcraft: Looking for a group», que celebra los 10 años de creación explorando historias inéditas. Igualmente, existe un mercado negro que mueve millones de dólares. Además, hay psicólogos que crean personajes dentro del WoW para hacer terapias online, famosos que han confesado jugar, personajes del videojuego que rinden homenaje a actores fallecidos como Robin Williams (seguidor incondicional del videojuego), bodas cibernéticas entre personajes, etc.

Se trata de un videojuego de suscripción en la que los jugadores pagan mensualmente para poder jugar. Al entrar en el videojuego el primer paso es elegir un reino o servidor, se tratan de mundos individuales en los que el usuario jugará. Seleccionado el mundo se debe elegir si formar parte de la Horda o de la Alianza, con esta elección el usuario determina si forma parte de los «malos» (Horda) o de los «buenos» (Alianza). Entre estos dos bandos no existe el contacto, sólo los miembros de una facción pueden interactuar entre ellos, comerciar, hablar, relacionarse y compartir hermandades. El siguiente paso es elegir la raza, la clase del personaje y darle nombre y aspecto. Con el personaje creado el jugador se introduce en 
el mundo y debe hacer que su barra de experiencia crezca para alcanzar los niveles superiores del juego. Este hito se consigue cumpliendo misiones y acumulando puntos. En los inicios del videojuego el nivel máximo era 60 , pero con el tiempo ha ido creciendo y en la actualidad el máximo nivel es 90 . A medida que se avanza en el juego, se batalla con otros jugadores y se completan misiones. El juego otorga a sus jugadores experiencia, objetos y dinero virtual para ir subiendo de categoría.

Existen hermandades o guilds, formadas por jugadores que comparten bancos de material, éstos son espacios virtuales donde incluyen sus pertenencias y adquisiciones en el juego para compartir con sus compañeros de hermandad. Además los miembros de una hermandad pueden comunicarse con más facilidad a través de programas externos como el teamspeak, y que compartir nombre y mismos objetivos.

El mundo de World of Warcraft es a tiempo real. Esto es: los días tienen 24 horas. De hecho existen actividades o festividades de la realidad que son trasladas al videojuego como Halloween, Navidad... se trata de un videojuegos donde los cambios en el mundo de Warcraft son continuos y no cesan.

Además el mundo de Warcraft también tiene una meteorología propia: puede llover, hacer sol... como en la vida real. Los jugadores desarrollan oficios seleccionados por ellos para mejorar sus habilidades. En las ciudades existen casas de subasta, peluquerías... En este tipo de videojuegos MMORPG es común que el videojuego no llegue a su fin con la muerte del personaje. Según el videojuego existen diferentes formas de hacer que el personaje resucite o reviva después de ser abatido en el videojuego.
Existen dos modalidades para jugar el World of Warcraft, la primera es PVP (player versus player) en la que los jugadores entran en el juego para luchar unos contra y la PVE (player versus environment) en la que los jugadores juegan contra el entorno, contra la máquina, contra el mundo creado por Blizzard y los personajes que la empresa ha creado. Ésta última es la versión más extendida.

Un punto importante del videojuego se produce cuando se logra alcanzar el nivel máximo y resulta posible hacer raids. Se tratan de misiones conjuntas con tus compañeros de hermandad para matar a un mismo boss (monstruo del juego). Las hermandades suelen organizarse jerárquicamente. Existe un rey líder que es el jefe y el que dirige a los jugadores en sus misiones. Al ser un juego en tiempo real, los jugadores deben conectarse a la misma hora para juntos ir a completar la misión que se han propuesto cumplir. Entre los miembros de una hermandad existen personajes de diferentes razas y habilidades que se complementan entre ellos para poder matar al enemigo y obtener recompensas. Este videojuego se desarrolla en una interfaz compleja en la que hay que saber aprovechar al máximo la pantalla para poder manejar al personaje aprovechando todas sus capacidades.

\section{Metodología.}

La metodología empleada parte de un producto audiovisual creado ad hoc en el marco de la investigación (concretamente, un documental que realiza un seguimiento detallado de un grupo de 5 jugadores del videojuego seleccionado). La pieza de 22 minutos de duración, pero con una grabación de más de 30 horas, registra la conducta de los protagonistas del producto audiovisual 
mientras juegan al videojuego y en situaciones de la vida cotidiana externas al videojuego. Además se ha hecho una aproximación al videojuego a través de una observación participante mientras se rodaba el documental, durante 4 meses.

Por otro lado se ha realizado un estudio descriptivo mediante un análisis del material grabado para la realización de producto audiovisual y entrevistas en profundidad a 4 expertos en videojuegos y educación, así como a educadores. Un total de 4 entrevistas realizadas a: Pere Marqués (DIM-UAB) maestro, licenciado en económicas y doctor en pedagogía (UAB), Pilar Lacasa (UAH) Catedrática de comunicación y educación de la Universidad Alcalá de Henares, investigadora en Utah (california) y en el instituto tecnológico de Massachusetts (MIT), José Luís Linaza (UCM) Doctor en psicología por la Universidad de Oxford y Licenciado en Filosofía y Letras por la universidad de Madrid (UCM), es catedrático en Psicología Evolutiva y de la Educación por la Universidad Autónoma de Madrid, y Begoña Gros (UB) Doctora en Pedagogía por la Universidad de Barcelona (UB). Desde1994 coordina el grupo de investigación «GrupF9» sobre el uso de los videojuegos en la enseñanza.

Este conjunto de herramientas metodológicas se centran en el estudio de los fenómenos sociales en el propio entorno natural en el que ocurren, dando importancia a la conducta humana. Se tratan de procesos de pensamiento situado (Gee, 2003), en los que los usuarios de videojuegos piensan, resuelven y aprenden los problemas mientras están jugando, en el contexto y con la gente que juegan en ese preciso momento. Este tipo de aprendizaje tiene lugar a través de la interacción con otros en un contexto de resolución de problemas auténtico. El producto audiovisual está grabado en los lugares habituales de juego de los protagonistas sin ningún tipo de alteración y las secuencias exteriores forman parte de la vida cotidiana de los jugadores.

En la investigación se analizan sus reacciones durante la participación en el videojuego y se intenta entender cómo los jugadores crean su propio mundo a partir de una investigación cualitativa, que ha permitido entrar dentro del proceso de construcción social del escenario del videojuego participando de las acciones que los propios jugadores desarrollan cuando juegan. Es decir, se ha participado desde dentro del juego en los chats y conversaciones de los usuarios como uno más para conocer las características sociales.

El material filmado durante el producto audiovisual, ha generado un total de 30 horas, en un proceso de grabación que duró 5 meses. El análisis del material (a través del minutado del mismo) se analizó siguiendo un esquema de ítems seleccionados previamente que son: Reflexiones, realidad vs ficción. Tipologías de roles - (role - playing). Resolución de problemas. Construcción de redes. (Socialización). Creatividad. (Creación de personajes). Valores educativos. Elementos negativos. Nuevas competencias, conocimientos y habilidades. Lenguaje propio y comunicación. El material recoge conversaciones entre los jugadores, entrevistas a los participantes, acciones de la vida cotidiana de los personajes que son analizadas en detalle para extraer las conclusiones objeto de la investigación.

Por todo ello, es importante destacar que se trata de una investigación experimental. De este modo, se aprovecha la inmersión en el videojuego World of Warcraft durante la 
grabación del documental y se utiliza la vivencia como parte de la investigación. Se trata de una observación participante en la que se llega a conocer en primera persona los efectos que el videojuego puede llegar a tener en los jugadores. Es una observación cualitativa (Hernández, Fernández \& Baptista, 2010) que supone una participación activa del observador, dejando de lado una mirada pasiva e implicándose en las situaciones manteniendo una actitud activa frente a las situaciones en las que se participa. Consiste en una metodología muy eficaz en el estudio de los videojuegos, ya que lo más importante para hablar de videojuegos es primero de todo conocerlos y haber jugado a ellos, como indica Pilar Lacasa en la entrevista que realizamos en la presente investigación.

Se complementa la metodología utilizada en esta investigación con cuestionarios realizados a jugadores y ex jugadores del videojuego de forma online que nos han permitido obtener datos estadísticos de forma rápida y concisa. Se realizaron un total de 220 encuestas, distribuidas mediante las redes sociales, de jugadores y ex jugadores de World of Warcraft.

Para completar la metodología, se realizaron entrevistas a expertos en materia de videojuegos, de educación y de alfabetización (citados con anterioridad), para otorgar un aporte científico y experimentado a los resultados de la investigación preguntándoles sobre si los videojuegos pueden ser educativos, sus posibles implicaciones en la educación formal y no formal, además de preguntas más específicas sobre el World of Warcraft y videojuegos MMORPG como sistemas de aprendizaje.

\section{Resultados.}

Como resultados generales, derivados de la investigación desarrollada, es posible afirmar en primer lugar y de forma más genérica que el empleo formativo de los videojuegos es muy amplio. Partiendo por las competencias y habilidades que los usuarios adquieren jugando, como por ejemplo, aprender a decodificar lenguajes digitales o aprender a dirigir y gestionar grupos con las Hermandades. Además se adquiere un nivel de inglés como idioma oficial del juego.

Los aspectos negativos también aparecen como resultados destacados. Casos de adicción producidos en ocasiones que se manifiestan en una confusión y sustitución de la realidad con la ficción consumiendo el tiempo libre y alejando al jugador de actividades más saludables.

La fantasía y creatividad es una característica explotada a través de estos videojuegos, en los que los jugadores dejan de ser ellos mismos para ser otras personas, una segunda vida virtual que en ocasiones puede llegar a ser demasiado real, permitiéndoles desconectar de la realidad y vivir experiencias que nunca conseguirían en la vida real.

Sobre las 22 encuestas destaca la conclusión de que los encuestados destacan haber adquirido a través del juego capacidades como el trabajo en equipo, la mejora de sus reflejos, la agilidad visual, nuevos lenguajes e idiomas y la habilidad de resolver problemas.

Para finalizar, los expertos afirmaron que la educación a través de los videojuegos es factible. Aunque no todos están de acuerdo en la inserción de esta herramienta en el aula, todos afirman que los jugadores adquieren competencias digitales y habilidades que 
contribuyen al desarrollo emocional de los usuarios y a la adquisición de competencias del ámbito de la alfabetización digital.

\section{Discusión.}

El trabajo desarrollado permite incidir en las amplias posibilidades de los videojuegos como recursos de gran potencial educativo. Concretamente, se refuerza la amplia variedad de posibilidades de formación y aprendizaje que introducen a pesar de no ser recursos diseñados para la educación.

Junto a ello, el estudio permite destacar un aspecto socializador y comunicativo de gran importancia en el desarrollo personal de los jugadores, estableciendo relaciones de diferente índole con otros jugadores. Este conjunto de habilidades ofrece la posibilidad de desarrollar habilidades y competencias del ámbito comunicativo de aplicación directa en la cotidianidad de los propios jugadores.

Otro aspecto destacable es el desarrollo de la creatividad que se convierte en un aspecto constante en el proceso del juego y que demanda de los usuarios la generación de estrategias, contenidos y otros tipos de recursos que demandan de grandes dosis de creatividad e innovación.

A lo anterior se suma el desarrollo de diversas habilidades tecnológicas que se van adquiriendo con el videojuego. Competencias que bien se pueden incluir dentro de los procesos de alfabetización digital y mediática. Entre estas destacan las acciones relacionadas con la búsqueda de recursos, el trabajo en equipo, aprendizaje de nuevos idiomas, etc. En todos los casos, se trata de tareas que los profesores enseñan en el aula, y que el videojuego enseña de forma informal a sus jugadores.
Por lo tanto, se pude concluir que nos encontramos ante un nuevo escenario educativo que construyen un conjunto de videojuegos que han sido catalogados como «no educativos». Se trata de un escenario por explotar y analizar en profundidad, que demanda de más y diversas investigaciones.

\section{Referencias bibliográficas.}

Aarseth, E. (2003, May) Playing reseacrh: methodological approaches to game análisis. Paper presented at the Digital Arts and Culture Conference, Melbourne, Australia.

Abt, C. C. (1987). Serious games. Lanham, MD: University Press of America.

Aranda, D. \& Sánchez, J. (2009). Aprovecha el tiempo y juega. Algunas claves para entender los videojuegos. Barcelona: UOC.

Castells, M. (2003). La galaxia Internet. Barcelona: Mondadori.

Echevarría, J. (2000). Avatares, realidad virtual y educación digital. Actas del I Congreso Internacional de Educación Digital (pp. 130-135). Bilbao.

Gardner, H. (1993). Inteligencias múltiples. La teoría en la práctica. Barcelona: Paidós.

Gee, J.P. (2003). What digital Games have to teach us. About learning and Literacy. New york: Palgrave Macmillan.

Gil, A. \& Vida, T. (2007). Els videojocs. Barcelona: UOC.

Gómez, D. (2001). Ideología y videojuegos. Revista en. red. ando. Recuperado de http:// w w w . 1 a $\mathrm{f}$ h . $\mathrm{i}$ n $\mathrm{f}$ o / staticViewPage.php?cat_ID=90\&idioma=ES

Haste, H. (2010). Ditizenship Education: A critical look at Contested Field. En L.R. Sherrod, J. Torney \& C.A. Flanagan (Eds.), Handbook of Research on Civic 
Engagement in Youth (pp.161-188). New Jersey; Jhon Wiley \& Sons.

Hernández, R., Fernández, C. F. \& Baptista, P. B. (2010). Metodología de la Investigación (5ªd.). México: McGraw Hill.

Joyanes, A. (1997). Cibersociedad: Los retos sociales ante un nuevo mundo digital. Madrid: McGraw Hill.

Koster, R. (2004). A theory od fun for game design. Scottsdale: Paraglyph Press.

Marqués, P. (2001). Los videojuegos. Departamento de Pedagogía Aplicada, Facultad de educación, UAB. Disponible online: peremarques.pangea.org/ videojue.htm

Maÿra, F. (2013) Entrevista realizada en el II congreso Internacional de Educación mediática y competencia digital, por el Gabinete de comunicación y educación. Disponible online: http://youtu.be/ QLjxbO4tYy8.

McGonigal, J. (2011). Reality Is Broken: Why Games Make Us Better and How They Can Change the World. New York, NY: Penguin.

Muros, B. (2011). El concepto de identidad en el mundo virtual: el «yo» on-line. Revista Electrónica Interuniversitaria de Formación del Profesorado, 37 (14-2), 49-56.

Pérez, J. M. \& Varis, T. (2012). Alfabetización mediática y nuevo humanismo. Barcelona: UOC.

Rheingold, H. (1996). La comunidad virtual: una sociedad sin fronteras. Barcelona: Gedisa.

Salen, K. \& Zimmerman, E. (2003). Rules of Game. Game Design Fundamentals. Cambridge: MIT Press.

Silva, A. (2010). El mundo relacional de la cibersociedad. Revista Estudios Culturales, 5, 89-106.
Thomas, D. \& Brown, J. S. (2007). The Play of imagination: Extending the Literary Mind. Games and Culture, 2, 149-172. doi: 10.1177/ 1555412007299458
Fecha recepción: 26-10-2015

Fecha evaluación: 13-11-2015

Fecha aceptación: 30/11/2015 